\title{
Birefringence dispersion in a quartz crystal retrieved from a channelled spectrum resolved by a fibre-optic spectrometer
}

\author{
Petr Hlubina*, Dalibor Ciprian \\ Department of Physics, Technical University Ostrava, 17. listopadu 15, \\ 70833 Ostrava-Poruba, Czech Republic
}

\begin{abstract}
We present a simple method for measuring the wavelength dependence of both the phase and group birefringence in a quartz crystal of known thickness. The method utilizes interference of polarized waves resolved by a fibre-optic spectrometer as a channelled spectrum (spectral fringes). The fringe order versus the precise position of the interference maximum in the spectrum is fitted to the approximate function, from which the phase birefringence as a function of wavelength is retrieved. We also determine the group birefringence dispersion. The functions measured in a range from 500 to $900 \mathrm{~nm}$ are compared with those resulting from the available dispersion relation, and very good agreement is confirmed.
\end{abstract}

Key words: spectral interference, birefringent crystal, fibre-optic spectrometer, channelled spectrum, birefringence dispersion, quartz crystal

PACS: $42.25 . \mathrm{Hz}, 42.25 . \mathrm{Lc}, 78.20 . \mathrm{Fm}$

* Tel.: +420-597-323-134; fax: +420-597-323-139.

Email address: petr.hlubina@vsb.cz (Petr Hlubina). 


\section{Introduction}

The phase and group birefringences and their dispersion, that is, the wavelength dependence, are the fundamental parameters and characteristics of anisotropic optical materials [1] used in optical devices such as wave plates, compensators, retarders, polarizers, etc. Spectral interferometry, which is based on the spectrally resolved interference of the polarization eigenvawes propagating through an anisotropic material and utilizes a broadband (white-light) source, is considered as one of the best tools to measure the phase and/or group birefringence dispersion in different anisotropic optical materials over a broad spectral range.

One of the most simple spectral interferometric techniques used to measure the phase birefringence dispersion is based on the observation of spectrally resolved interference fringes (channelled spectrum) in a set-up with an anisotropic optical material placed between a polarizer and an analyser [2,3]. The channelled spectrum originates from the interference of the polarization eigenvawes propagating through the anisotropic material illuminated with a collimated beam. The technique is usable for both uniaxial and biaxial anisotropic crystals and it enables one to determine the phase birefringence dispersion from the positions of maxima in a channelled spectrum. The fringe order as a function of wavelength is fitted to a prescribed function $[2,3]$ from which the phase birefringence dispersion is retrieved for the crystal of known thickness. Similarly, the phase birefringence of a prescribed dispersion can be measured in a set-up with a Mach-Zehnder interferometer using fitting the measured spectrum to the theoretical one [4].

White-light spectral interferometry with a standard Michelson interferometer is also usable for measuring the group birefringence dispersion [5], the refractive index dispersion [6] or the group delay dispersion [7] in uniaxial anisotropic crystals. In the first case, the tandem configuration of the Michelson interferometer and the birefringent crystal (placed between a polarizer and an analyser) is used, while in the remaining cases the crystal is placed into a test arm of the interferometer. In the first two cases, the group dispersion is retrieved from the dependence of the equalization wavelength on the path difference adjusted in the interferometer. The equalization wavelength corresponds to a stationary-phase point which appears in the recorded spectral interferogram when the overall group optical path difference in the interferometer is close to zero and the spectral fringes have nearly infinite period. In some of the measurements $[5,6]$, a portable miniature fibre-optic spectrometer has emerged as a modern, low-cost and powerful tool of spectral analysis.

In this paper, a simple method for measuring the wavelength dependence of both the phase and group birefringence in a quartz crystal of known thick- 
ness is presented. The technique, which is based on recording of a channelled spectrum by a fibre-optic spectrometer, is used to determine the precise positions of interference maxima. The fringe order versus the wavelength of the interference maximum is fitted to a prescribed function from which the phase birefringence as a function of wavelength is retrieved. The phase birefringence dispersion in the quartz crystal measured in a range from 500 to $900 \mathrm{~nm}$ with a relative error of $7 \times 10^{-4}$ agrees well with that obtained from the dispersion relation proposed by Ghosh [8]. We also determine the group birefringence dispersion in the quartz crystal and confirm very good agreement with the available data.

\section{Experimental method}

Consider the experimental set-up shown in Fig. 1 comprising a white-light source (WLS), a collimating lens (L1), a polarizer (P), a uniaxial birefringent crystal (BC) of thickness $t$, an analyser (A), a lens (L2), a read optical fibre (ROF) and a spectrometer. The crystal is characterized by the wavelengthdependent birefringence $B_{\mathrm{f}}(\lambda)$ defined as

$$
B_{\mathrm{f}}(\lambda)=n_{e}(\lambda)-n_{o}(\lambda)
$$

where $n_{e}(\lambda)$ and $n_{o}(\lambda)$ are the phase refractive indices of the extraordinary and ordinary eigenwaves, respectively. For convenience, the $z$ axis is chosen along the propagation direction of the collimated beam through the birefringent crystal and the $x$ axis is along the vertical direction. For the birefringent crystal with the optic axis at $90^{\circ}$ with respect to the $x$ axis, the polarization directions of eigenwaves are at $0^{\circ}$ and at $90^{\circ}$ with respect to the $x$ axis. The transmission azimuth of the polarizer is adjusted at $45^{\circ}$ with respect to the $x$ axis of the $\mathrm{BC}$ so both polarization eigenwaves are excited equally in the $\mathrm{BC}$. The two polarization eigenwaves propagating through the $\mathrm{BC}$ are mixed with the analyser and their interference is resolved by the spectrometer as a channelled spectrum. The transmission azimuth of the analyser is adjusted at $45^{\circ}$ with respect to the $x$ axis of the BC. The spectral intensity recoded by the spectrometer of a Gaussian response function can be represented in the form $[5]$

$$
I(\lambda)=I_{0}(\lambda)\left\{1+V_{\mathrm{R}}(\lambda) \cos \left[(2 \pi / \lambda) B_{\mathrm{f}}(\lambda) t\right]\right\},
$$

where $I_{0}(\lambda)$ is the reference (unmodulated) spectrum and $V_{\mathrm{R}}(\lambda)$ is a visibility term given by

$$
V_{\mathrm{R}}(\lambda)=\exp \left\{-\left(\pi^{2} / 2\right)\left[G_{\mathrm{f}}(\lambda) t \Delta \lambda_{\mathrm{R}} / \lambda^{2}\right]^{2}\right\}
$$


where $G_{\mathrm{f}}(\lambda)$ is the group birefringence given by

$$
G_{\mathrm{f}}(\lambda)=B_{\mathrm{f}}(\lambda)-\lambda \frac{\mathrm{d} B_{\mathrm{f}}(\lambda)}{\mathrm{d} \lambda}=-\lambda^{2} \frac{\mathrm{d}\left[B_{\mathrm{f}}(\lambda) / \lambda\right]}{\mathrm{d} \lambda}
$$

and $\Delta \lambda_{\mathrm{R}}$ is the width of the response function of the spectrometer. To resolve a channelled spectrum (spectral fringes) in a spectral range from $\lambda_{1}$ to $\lambda_{2}$, the thickness of the $\mathrm{BC}$ with normal birefringence dispersion must satisfy the condition $t<t_{\max }$, where

$$
t_{\max }=\frac{\lambda_{1}^{2}}{G\left(\lambda_{1}\right) \Delta \lambda_{\mathrm{R}}} .
$$

We can resolve in the recorded channelled spectrum a large number of spectral fringes. The interference maximum (a bright fringe) satisfies the relation

$$
B_{\mathrm{f}}(\lambda) t=m \lambda,
$$

where $m$ is the order of interference of the fringes. After counting $i$ bright spectral fringes in the direction of shorter wavelengths, Eq. (6) can be written as

$$
B_{\mathrm{f}}(\sigma) t \sigma=m+i
$$

where $\sigma=1 / \lambda$ is the wavenumber.

The wavenumber dependence of the phase birefringence $B_{\mathrm{f}}(\sigma)$ in the $\mathrm{BC}$ can be well approximated by a modified Cauchy dispersion formula [9]

$$
B_{\mathrm{f}}(\sigma)=A+B \sigma^{2}+\frac{C}{\sigma^{2}}
$$

where $A, B$ and $C$ are constants characterizing the birefringent crystal. The last term in Eq. (8) extends a two-term Cauchy formula [1] to better characterize the group birefringence dispersion in the BC over a broad spectral range. On substituting from Eq. (7) into Eq. (8), we obtain

$$
a \sigma+b \sigma^{3}+\frac{c}{\sigma}=m+i
$$

where $a=A t, b=B t$ and $c=C t$. By a least-squares fitting of Eq. (9), the constants $a, b, c$ and $m$ are determined, and knowing the thickness $t$, the wavelength dependence of the phase birefringence $B_{\mathrm{f}}(\lambda)$ can be deduced from Eq. (8). This approach is much simpler in comparison with our previous 
approach [10] because there is no need to retrieve a relative phase function from the channelled spectrum.

\section{$3 \quad$ Experimental set-up}

The experimental set-up used for measurement of dispersion of the phase birefringence $B_{\mathrm{f}}(\lambda)$ in a $\mathrm{BC}$ is shown schematically in Fig. 1. It consists of a halogen lamp (HL-2000, Ocean Optics), a launching fibre, a collimating lens, a Glan-Taylor calcite polarizer and analyser (Thorlabs), a quartz crystal under test, a microscope objective $(10 \times / 0.30)$, a micropositioner, a fibre-optic spectrometer (S2000, Ocean Optics), an A/D converter and a personal computer. The quartz crystal under test consists of two polished surfaces, parallel with a high precision to the optic axis of the crystal. The crystal is placed into the set-up in such a way that the collimated beam is incident on the surfaces perpendicularly and the orientation of the optic axis is shown in Fig. 1. The thickness of the crystal is $t=(4010 \pm 1) \mu \mathrm{m}$.

The fibre-optic spectrometer S2000 of an asymmetric crossed Czerny-Turner design with the input and output focal lengths of 42 and $68 \mathrm{~mm}$, respectively, has a spectral operation range from 350 to $1000 \mathrm{~nm}$ and includes a diffraction grating with 600 lines per millimetre, a 2048-element linear CCD-array detector with a Schott glass long-pass filter, a collection lens and a read optical fibre. The wavelength of the spectrometer is calibrated so that a third-order polynomial relation between pixel number and wavelength is used. The spectrometer resolution is in our case given by the effective width of the light beam from a core of the read optical fibre: a $50 \mu \mathrm{m}$ core diameter corresponds a Gaussian response function with $\Delta \lambda_{\mathrm{R}}=3 \mathrm{~nm}$ [11]. Spectrometer sensitivity is, at given light conditions, affected by the spectrometer integration time: it can easily be varied under software control.

\section{Experimental results and discussion}

We demonstrate the ability of our method in measuring the phase birefringence $B_{\mathrm{f}}(\lambda)$ in the quartz crystal of known thickness for which the channelled spectrum shown in Fig. 2 was recorded. It is clearly seen from Fig. 2 that a large number of the spectral interference fringes (namely 35) of a sufficiently high visibility are resolved. Moreover, it is clear that for a thicker crystal the number of spectral fringes will increase and the shortest wavelength $\lambda_{1}$ at which the spectral fringes have the lowest visibility will shift, as indicates Eq. (5), to the long wavelength range. In other words, the number of fringe orders resolved in the recorded channelled spectrum, which is related to the accuracy 
of the measurement, is affected not only by the width of the source spectrum, but also by the thickness of the birefringent crystal.

The procedure used to retrieve the phase birefringence $B_{\mathrm{f}}(\lambda)$ from the recorded channelled spectrum consists of two steps. In the first step, the wavelengths of interference maxima are determined for the recorded channelled spectrum $I(\lambda)$. The spectral interference fringes are numbered in such a way that the fringe order increases in the direction of shorter wavelengths. Figure 3 shows by the crosses the dependence of the fringe order on the wavelength obtained from the channelled spectrum shown in Fig. 2. In the second step, a leastsquares fit of Eq. (9) is used to the dependence which gives the constants $a, b, c$ and $m$. Figure 3 shows the results of the fit by the solid line. Then the constants $a, b, c$ and the known thickness $t$ give the constants $A, B, C$ of the dispersion relation (8), which is used to determine to the wavelength dependence of the phase birefringence $B_{\mathrm{f}}(\lambda)$. In Fig. 4 are shown by the crosses the values obtained by the above procedure for the quartz crystal under test. The same figure also shows the phase birefringence $B_{\mathrm{f}}(\lambda)$ resulting from the Sellmeier-like form of the dispersion relation for the refractive indices $n_{o}(\lambda)$ and $n_{e}(\lambda)$ of the quartz crystal proposed by Ghosh [8]:

$$
n_{o, e}^{2}(\lambda)=A_{o, e}+\frac{B_{o, e} \lambda^{2}}{\lambda^{2}-C_{o, e}}+\frac{D_{o, e} \lambda^{2}}{\lambda^{2}-F_{o, e}} .
$$

If the wavelength $\lambda$ is in microns, the dispersion coefficients at room temperature are as follows: $A_{o}=1.28604141, B_{o}=1.07044083, C_{o}=1.00585997 \times$ $10^{-2}, D_{o}=1.10202242$ and $F_{o}=100 ; A_{e}=1.28851804, B_{e}=1.09509924$, $C_{e}=1.02101864 \times 10^{-2}, D_{e}=1.15662475$ and $F_{e}=100$.

We clearly see that there is very good agreement between these dependences, which have slightly different dispersion slopes. From the measured phase birefringence $B_{\mathrm{f}}(\lambda)$ also the group birefringence $G(\lambda)$ given by Eq. (4) can be evaluated. This dependence is shown in Fig. 4 by the upper crosses together with the solid line, which corresponds to the group birefringence $G(\lambda)$ evaluated from the group refractive indices $N_{o}(\lambda)$ and $N_{e}(\lambda)$ in the quartz crystal given by [6]

$$
N_{o, e}(\lambda)=n_{o, e}(\lambda)+\frac{\lambda^{2}}{n_{o, e}(\lambda)}\left\{\frac{B_{o, e} C_{o, e}}{\left(\lambda^{2}-C_{o, e}\right)^{2}}+\frac{D_{o, e} F_{o, e}}{\left(\lambda^{2}-F_{o, e}\right)^{2}}\right\} .
$$

As it is clearly seen from Fig. 4, the measured and approximated group birefringences differ each other at shorter wavelengths. The difference between them, which is in general dependent on the fitting function [10], is in part caused by the approximation (8) of the phase birefringence dispersion $B_{\mathrm{f}}(\lambda)$ we used. 
We can estimate a precision of the measurement of the phase birefringence. If the wavelength $\lambda$ of the maximum is known with a precision of $\delta \lambda$ and the thickness $t$ of the birefringent crystal is known with a precision of $\delta t$, the relative error of the measurement of the phase birefringence is given by the following formula:

$$
\frac{\delta B_{\mathrm{f}}}{B_{\mathrm{f}}}=\sqrt{\left(\frac{\delta \lambda}{\lambda}\right)^{2}+\left(\frac{\delta t}{t}\right)^{2}} .
$$

For the error $\delta \lambda=0.32 \mathrm{~nm}$, which is given by the wavelength difference corresponding to adjacent pixels of the spectrometer linear CCD-array detector, and for the precision $\delta t=1 \mu \mathrm{m}$, the relative error of the measurement of the phase birefringence is approximately $7 \times 10^{-4}$. Higher measurement precisions can be achieved using the spectrometer with the smaller wavelength difference of adjacent pixels (e.g. USB4000, Ocean Optics [12]) and/or a thicker crystal.

\section{Conclusions}

We used a simple white-light spectral interferometric technique employing a fibre-optic spectrometer to measure the birefringence dispersion in a quartz crystal over a wide spectral range (500 to $900 \mathrm{~nm}$ ). From a channelled spectrum, which originates from the interference of the polarization eigenvawes propagating through the crystal illuminated with a collimated beam, the positions of the interference maxima were determined. The fringe order versus the precise position of the interference maximum in the spectrum was fitted to a prescribed function, from which the phase birefringence dispersion was retrieved for known thickness of the crystal. We confirmed that the phase birefringence dispersion in the quartz crystal measured with a relative error of $7 \times 10^{-4}$ agrees well with that obtained from the dispersion relation proposed by Ghosh [8]. Furthermore, we determined the group birefringence dispersion in the quartz crystal and confirmed very good agreement with the available data.

The results obtained serve as an illustration of the feasibility of a simple and cost-effective measurement technique based on the resolving of a channelled spectrum by using a fibre-optic spectrometer. It allows one to determine both the phase and group birefringence dispersion in a uniaxial crystal of known thickness. The use of the technique can be extended for measuring the phase and group birefringence dispersion in polarization-maintaining fibres. 


\section{Acknowledgment}

The research has been partially supported by the Ministry of Education, Youth and Sports of the Czech Republic through grant MSM6198910016, by the Grant Agency of the Czech Republic through grant P102/11/0675 and the regional grant CZ.1.05/2.1.00/01.0040. 


\section{References}

[1] M. Born, E. Wolf, Principles of Optics, seventh ed., Cambridge University Press, Cambridge, 1999.

[2] M. Medhat, S.Y. El-Zaiat, Opt. Commun. 141 (1997) 145.

[3] M. Emam-Ismail, Opt. Las. Technol. 41 (2009) 615.

[4] H. Delbarre, C. Przygodzki, M. Tassou, D. Boucher, Appl. Phys. B 70 (2000) 45.

[5] P. Hlubina, Opt. Commun. 251 (2005) 367.

[6] P. Hlubina, D. Ciprian, L. Knyblová, Opt. Commun. 269 (2007) 8.

[7] W. Yang, J. Li, F. Zhang, Y. Zhang, Z. Zhang, Opt. Commun. 281 (2008) 679.

[8] G. Ghosh, Opt. Commun. 163 (1999) 95.

[9] S.Y. El-Zaiat, Opt. Las. Technol. 34 (2002) 15.

[10] P. Hlubina, D. Ciprian, Opt. Lett. 35 (2010) 1566.

[11] P. Hlubina, I. Gurov, V. Chugunov, J. Mod. Opt. 50 (2003) 2067.

[12] Spectrometers, Catalog 07, Ocean Optics Inc., Dunedin 2007, 14. 


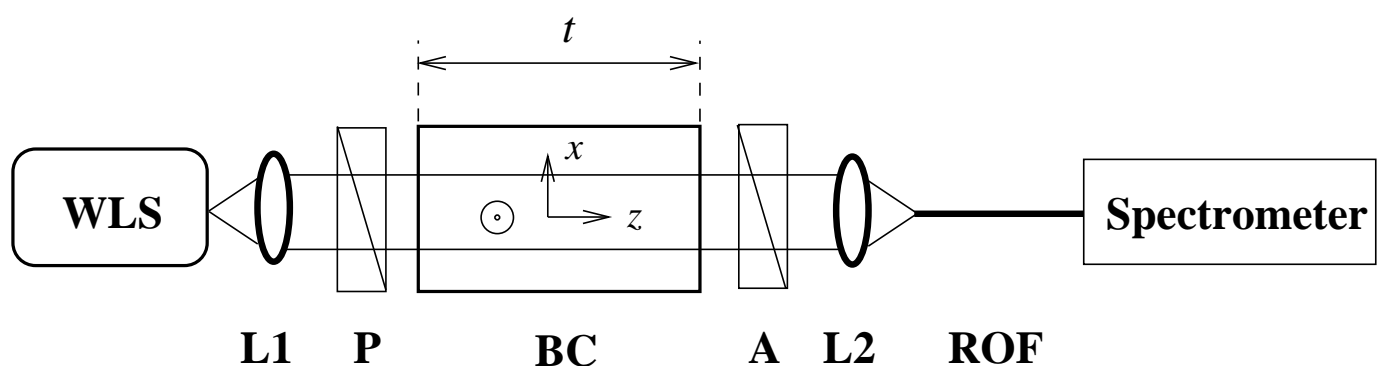

Fig. 1. Experimental set-up to record a channelled spectrum for a uniaxial birefringent crystal. 


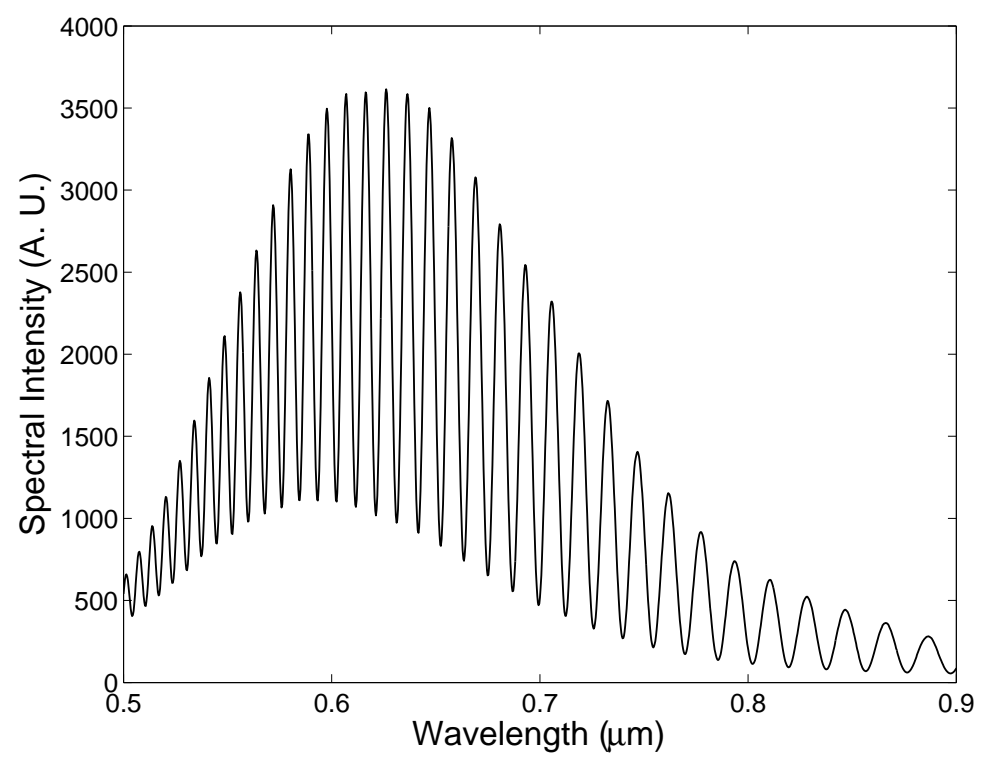

Fig. 2. Channelled spectrum recorded by a fibre-optic spectrometer for a quartz crystal of thickness $t=(4010 \pm 1) \mu \mathrm{m}$.

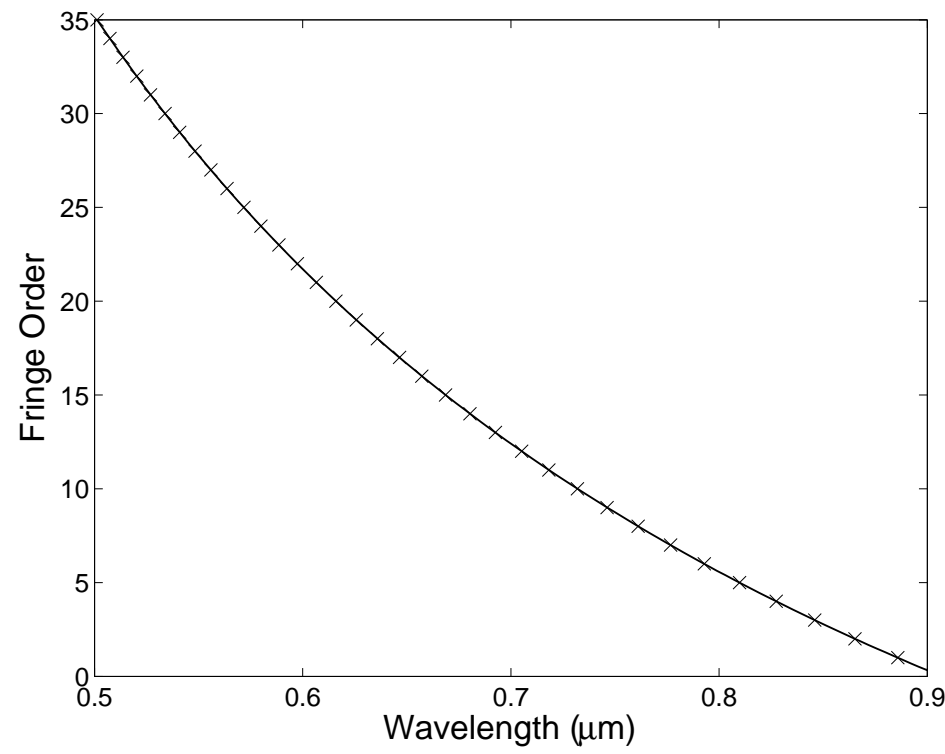

Fig. 3. Fringe order as a function of wavelength retrieved from the channelled spectrum shown in Fig. 2. The solid line corresponds to a fit. 


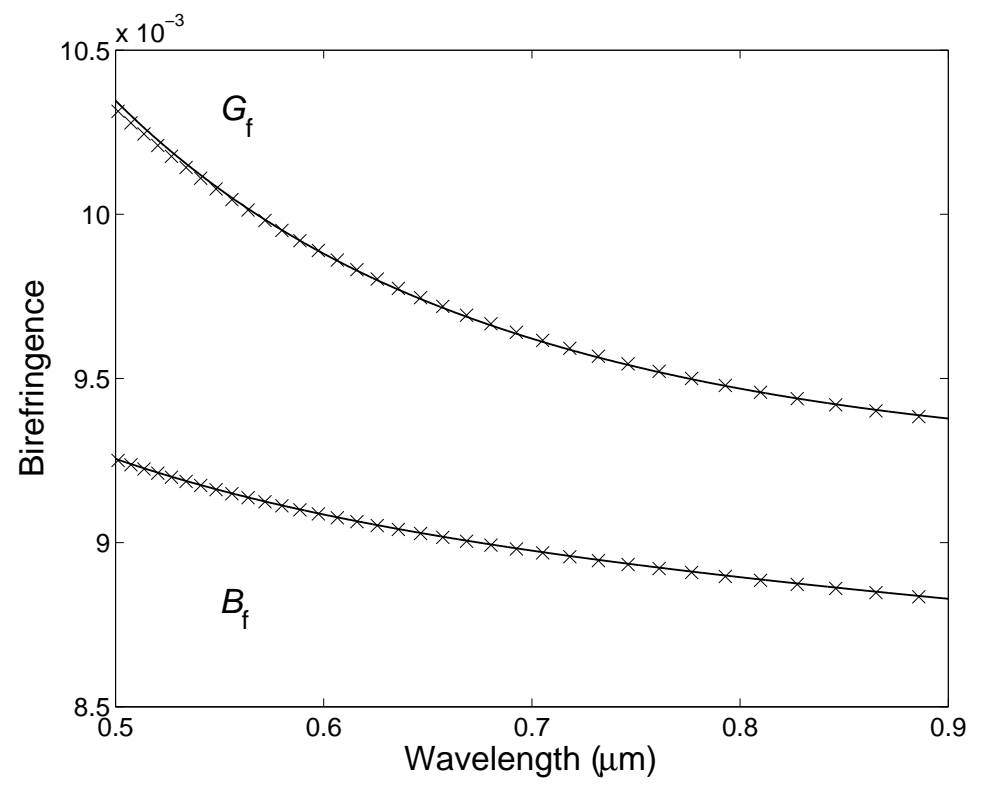

Fig. 4. Retrieved phase and group birefringences, $B_{\mathrm{f}}(\lambda)$ and $G_{\mathrm{f}}(\lambda)$, for a quartz crystal (crosses) compared with those given by the dispersion relation. 